\title{
Indirect measurement of pinch and pull forces at the shaft of laparoscopic graspers
}

\author{
John J. van den Dobbelsteen • Ruben A. Lee • \\ Maarten van Noorden · Jenny Dankelman
}

Received: 4 May 2011/Accepted: 5 January 2012/Published online: 19 January 2012

(c) The Author(s) 2012. This article is published with open access at Springerlink.com

\begin{abstract}
The grasping instruments used in minimally invasive surgery reduce the ability of the surgeon to feel the forces applied on the tissue, thereby complicating the handling of the tissue and increasing the risk of tissue damage. Force sensors implemented in the forceps of the instruments enable accurate measurements of applied forces, but also complicate the design of the instrument. Alternatively, indirect estimations of tissue interaction forces from measurements of the forces applied on the handle are prone to errors due to friction in the linkages. Further, the force transmission from handle to forceps exhibits large nonlinearities, so that extensive calibration procedures are needed. The kinematic analysis of the grasping mechanism and experimental results presented in this paper show that an intermediate solution, force measurements at the shaft and rod of the grasper, enables accurate measurements of the pinch and pull forces on tissue with only a limited number of calibration measurements. We further show that the force propagation from the shaft and rod to the forceps can be approximated by a linear two-dimensional function of the opening angle of the grasper and the force on the rod.
\end{abstract}

Keywords Health care Human performance . Medical simulation - Tactile device

J. J. van den Dobbelsteen $(\bowtie) \cdot$ R. A. Lee · M. van Noorden . J. Dankelman

Department of BioMechanical Engineering, Faculty of Mechanical, Maritime and Materials Engineering, Delft University of Technology, Mekelweg 2, 2628 CD Delft, The Netherlands

e-mail: j.j.vandendobbelsteen@tudelft.nl

\section{Introduction}

Accurate control of tissue grasping forces is an essential component of many surgical activities. An inability to control these forces is associated with slippage of the tissue when the forces are too low and with tissue damage when the forces are too high $[2,7]$. When there is direct contact between tissue and the surgeon's fingers, mechanoreceptors in the skin and muscles provide reliable information about the applied forces and enable optimal surgical performance $[4,9]$. The instruments required for minimally invasive surgery (MIS) eliminate this direct contact, reducing the quality of the force feedback to the surgeon [3]. This complicates the handling of the tissue and correctly applying the forces necessary, thereby enlarging the risk of tissue damage [6]. This has motivated researchers to explore alternatives to enable the surgeon to assess the forces applied on the tissue.

One possibility to assess applied forces is to implement force-sensing capability in the instruments. Previous research has explored direct and indirect force-sensing methods using laparoscopic instruments equipped with force sensors. In the direct approach, the sensors are placed in the forceps of the instrument for force measurements at the location of interest $[1,8,10,12,14,15]$. Indirect force sensing is typically accomplished by placing sensors at the handle of the instrument, remote from the site where the tool interacts with the tissue [5]. Both approaches have their own benefits and drawbacks. Incorporating sensors in the forceps enables highly accurate measurements of the forces applied on the tissue. However, the design of the required miniature structures is complex and involves major modifications of the instruments. These instruments are, therefore, difficult to produce and expensive.

Important advantages of the indirect approach are that all electronics are isolated from the patient and that it is 
suitable for a large variety of standard instruments. Indirect measurements may, however, lead to inaccurate results. The transmission of grasping forces from the handle to the tip of the tool is realized through a grasping mechanism that is actuated by the inner metal rod, which is mechanically coupled to the outer shaft and the handle. The force transmission depends on the geometry of the linkages between the handle, rod and grasping mechanism, and the various kinematic parameters of each component. Nonlinearities in the kinematics of the linkages complicate computing the grasping force exerted on the tissue from the input force at the handle [11]. Moreover, the internal friction forces in the joints of the mechanisms are not invariant, but a function of both the opening angle of the forceps and the force on the inner rod [13]. Hence, an extensive calibration procedure is needed for the transformation of the forces exerted on the handles to tissue forces.

Based on the previous work in this area, along with the disadvantages of these previous surgical tools, we propose an intermediate solution for the measurement of tissue interaction forces. By placing force sensors on the rod and shaft, as opposed to placing sensors on the handles, the accuracy of indirect measurements can be improved. Bypassing the linkage between handle and rod will reduce the distorting effects of friction and ease the identification of the input and output relationships. The theoretical analysis of the basic mechanics of standard graspers presented in this paper shows that the relationship between the opening angle of the grasper and the force transmission of the grasping mechanism can be described with sufficient accuracy by a linear function for a large variety of configurations of the mechanism. In an experimental study, we further show that the force transmission from the rod to the forceps of the instrument can be approximated with a linear two-dimensional function of the opening angle of the grasper and the force on the rod.

\section{Methods}

The first part of "Methods" describes a basic model that can be used to compute the pinch and pull force on the grasped object from the forces applied on the grasping mechanism of the laparoscopic tool. In the second part, we describe the experimental methods that were used to measure the force transmission of a standard laparoscopic grasper.

\section{Basic relationships}

Most laparoscopic graspers use more or less the same rhombus linkage. The designs generally consist of a handle mechanism, a transmission link (i.e., the rod) and a four-bar mechanism to actuate the forceps. The exact geometric parameters may vary from brand to brand but the basic principle of transferring forces from the handle into a grasping force is the same. The shaft is fixed to one pivoting point of a pair of links, a four-bar mechanism, and to the rigid part of the handle mechanism. A rotation of the handle translates the rod through the shaft. The rod then drives the four-bar mechanism to enable opening or closing of the forceps of the grasper. A schematic representation of the grasper is shown in Fig. 1.

Two forces can be transferred from the handle to the forceps: one acting on the shaft and the other acting on the inner rod. Referring to Fig. 2, we describe how these forces result in a pinching and pulling force onto the grasped object.

For simplicity, we first assume that there is no friction in the joints of the four-bar mechanism and that the pinching force is equal on both sides of the forceps due to the symmetry of the rhombus linkage. We further assume that the friction force between the forceps and the object is large enough to prevent slippage of the object during pinching and pulling.

Consider the situation when only a pinching force and no pulling force is applied. When there are no other forces
Fig. 1 Schematic representation of a standard laparoscopic grasper. 1 shaft, 2 rod, 3 handle, 4 forceps

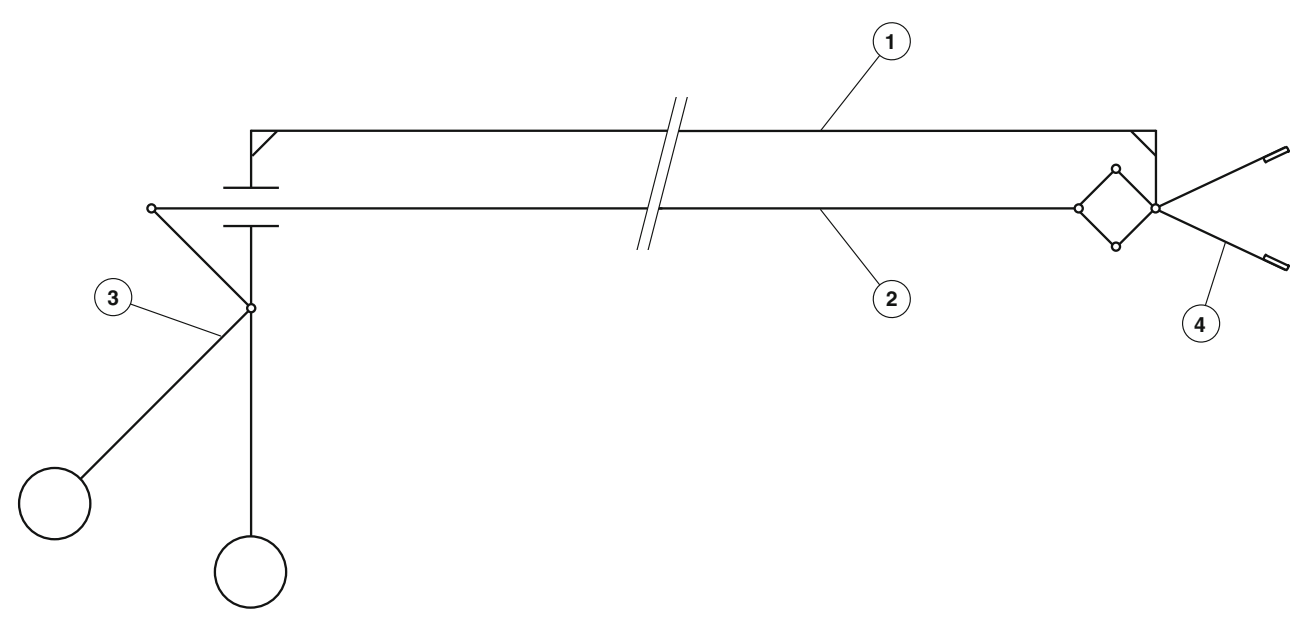




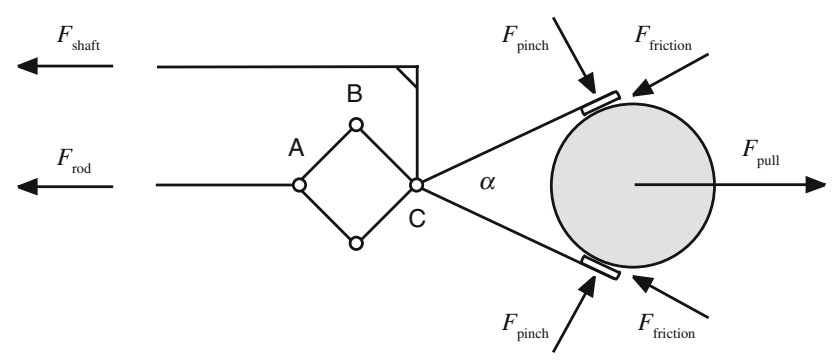

Fig. 2 Schematic representation of the rhombus linkage

working on the tool and the system is at rest (not accelerating), then:

$\sum F=0$

$F_{\text {shaft }}=-F_{\text {rod }}$

$F_{\text {pinch }}=\frac{1}{2} \cdot \lambda \cdot F_{\text {rod }}$

where $\lambda$ is the force transmission coefficient, which contains the complete geometry of the linkage.

When we consider the situation of combined pinching and pulling, the pulling force becomes:

$F_{\text {pull }}=F_{\text {shaft }}+F_{\text {rod }}$

and the pinching force will then be equal to:

$F_{\text {pinch }}=\frac{1}{2} \cdot \lambda \cdot\left(F_{\text {pull }}-F_{\text {shaft }}\right)$

For this situation, Eqs. 4 and 5 can be used to determine the pulling and pinching force applied onto the object when the forces on the rod and shaft and $\lambda$ are known.

\subsection{Modeling of force transmission $\lambda$}

The force transmission coefficient $\lambda$ is not constant, but is a function of the opening angle $\alpha$ of the forceps. The exact properties of the relation between $\lambda$ and $\alpha$ depend on the geometry of the rhombus linkage. For our idealized, symmetrical configuration of the four-bar mechanism, the theoretical relation between them can easily be derived. Here, we briefly mention the basic relations to compute the force transmission of the four-bar mechanism. A more extensive analysis of the force propagation from handle to forceps is given by [11].

A kinematic diagram of the four-bar mechanism is presented in Fig. 3a. The links between the joints A, B and $\mathrm{C}$ are of equal length. Angle $\beta$ is the angle between these links and the translating rod. Angle $\beta$ can be derived from the opening angle $\alpha$ and is defined by:

$\beta=\alpha+\beta_{0}$

Figure $3 b$ and $c$ displays the forces generated on the joints $\mathrm{A}, \mathrm{B}$ and $\mathrm{C}$ when a force is exerted on the rod and the
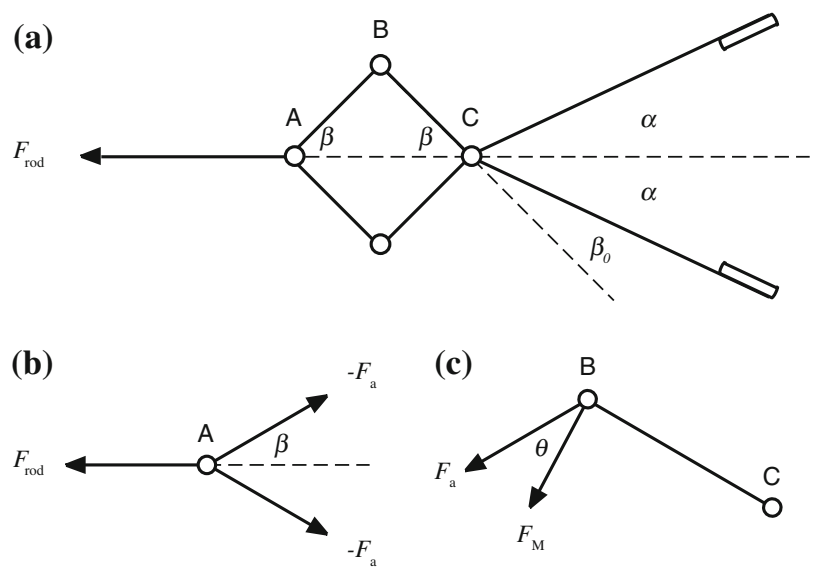

Fig. 3 Kinematics and force propagation of a four-bar mechanism joints are not moving relative to each other (i.e., the angles between the links are invariant when holding an incompressible object). The force $F_{a}$ on the link $\mathrm{AB}$ can be written as a function of $F_{\text {rod }}$ and the angle $\beta$ :

$F_{a}=\frac{F_{\text {rod }}}{2 \cdot \cos (\beta)}$

The force $F_{a}$ creates an effective force $F_{M}$ perpendicular to the link $\mathrm{BC}$, which causes a moment about the fixed pivot point $\mathrm{C}$.

$F_{M}=F_{a} \cdot \cos (\theta)$

where the angle $\theta$ can be derived from the angle $\beta$ as:

$\theta=\frac{\pi}{2}-2 \beta$

Using the Eqs. 7, 8 and 9, we can write the relationship between $F_{M}, F_{\text {rod }}$ and $\beta$ as:

$F_{M}=F_{a} \cdot \sin (2 \beta)$

$F_{M}=\frac{F_{\text {rod }}}{2 \cdot \cos (\beta)} \cdot \sin (2 \beta)$

$F_{M}=F_{\text {rod }} \cdot \sin (\beta)$

Equation 12 shows that for this idealized configuration, there is a simple sinusoidal relationship between the opening angle of the forceps and the force transmission $\lambda$. The exact force transmission is further determined by the constant ratio of the length of the link BC and the length of the forceps (assuming that the object is always grasped with the tip of the instrument) and the linear relationship between angle $\beta$ and the opening angle $\alpha$.

\subsection{Linearization of force transmission $\lambda$}

The basic model presented above shows that there is a nonlinear relationship between the opening angle of the grasper and the force transmission from the rod to the forceps. Nevertheless, in this section we show that this relationship 
can be described with sufficient accuracy by a linear function.

Equation 12 can be used to compute the force transmission coefficient for various opening angles $\alpha$ when angle $\beta_{0}$ is known. Angle $\beta_{0}$ is the angle between link BC and the forceps and equals angle $\beta$ when the grasper is fully closed (Eq. 6). If we assume a typical angle of $15^{\circ}$, then the force transmission coefficient for opening angle $\alpha$ can be obtained (Eq. 6). We further assume that the useful range of opening angles varies between $0^{\circ}$ and $30^{\circ}$ for each of the forceps of the graspers.

Figure 4 displays the force transmission coefficients for the force $F_{a}$ on link $\mathrm{AB}$ and the effective force $F_{M}$ on link $\mathrm{BC}$, as a function of opening angle $\alpha$. The relationship between coefficients for $F_{M}$ and $\alpha$ is roughly linear between this range of opening angles. Using leastsquares linear regression, this relationship can be described as:

$\lambda=0.015 \alpha+0.269$

The average absolute error of this approximation is 0.004 with a standard deviation of 0.002 showing that within the useful range of opening angles, a linear function describes the force transmission with sufficient accuracy.

In the analysis above, we assumed that the length of the links of the four-bar mechanism was equal. However, the exact geometry of the mechanism varies across different graspers, while the sinusoidal relationship only holds for our idealized grasper. Given the geometrical triangle bounded by the link $\mathrm{AB}$, link $\mathrm{BC}$ and the possible distances between joint $\mathrm{A}$ and $\mathrm{C}$, we can obtain the force transmission coefficients for other configurations using the law of cosines.

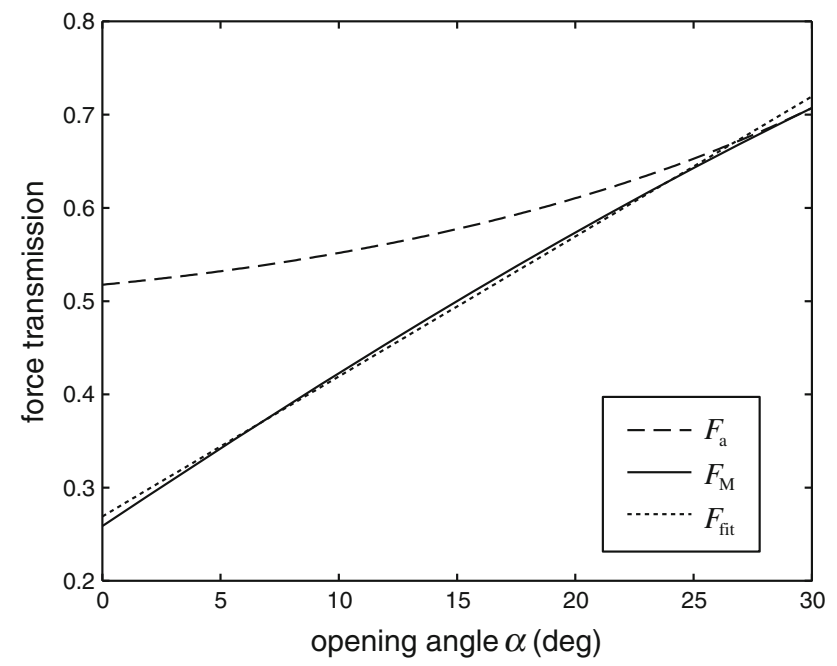

Fig. 4 Force transmission coefficients. The dotted line represents a linear fit to sinusoidal relationship between the force on the rod and the effective force on the link that drives the forceps
If we define $r$ as the ratio between length $a$ of link $\mathrm{AB}$ and the length of link $\mathrm{BC}$, and $c$ as the distance between $\mathrm{A}$ and $\mathrm{C}$, the following equations can be developed:

$F_{a}=\frac{F_{\mathrm{rod}} \cdot c \cdot a}{a^{2}-r^{2} \cdot a^{2}+c^{2}}$

$\theta=\sin ^{-1} \frac{a^{2}+r^{2} \cdot a^{2}-c^{2}}{2 \cdot r \cdot a^{2}}$

Figure 5 displays the force transmission coefficient as a function of opening angle $\alpha$ for a range of configurations of the four-bar mechanism. For this analysis, we varied the values of $r$ between 0.5 and 1.3 and $\beta_{0}$ was set to $15^{\circ}$.

For each line in Fig. 5, we performed a least-squares linear regression to see whether these functions can be approximated by a linear function with sufficient accuracy. Figure 6 displays the average absolute linearization errors of these approximations for each of the ratios $r$. The error approaches zero at a ratio of about 1.1, showing that the relationship between $\alpha$ and $\lambda$ is linear for this configuration. When the length of link BC becomes much larger than the length of link $\mathrm{AB}$, the error increases rapidly. Nevertheless, for all of these reasonable configurations the linearization errors are small. These results show that linearity between $\lambda$ and $\alpha$ can be assumed for a large diversity of configurations of the four-bar mechanism.

\subsection{Force transmission and internal friction}

The above theoretical analysis shows that the pinching and pulling force of a laparoscopic grasper can be computed from the forces applied on the rod and the shaft, even when the exact geometry of the rhombus link is not known. A simple linear function can be used to describe the relationship between the opening angle $\alpha$ and force transmission $\lambda$ with sufficient accuracy. However, so far we have

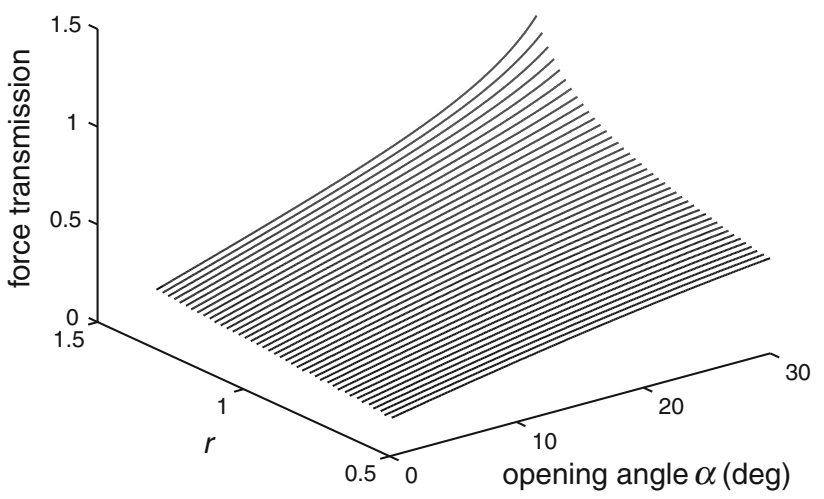

Fig. 5 Force transmission coefficients. Coefficients as a function of opening angle of the grasper for different ratios $r$ between the lengths of the links of the four-bar mechanism 


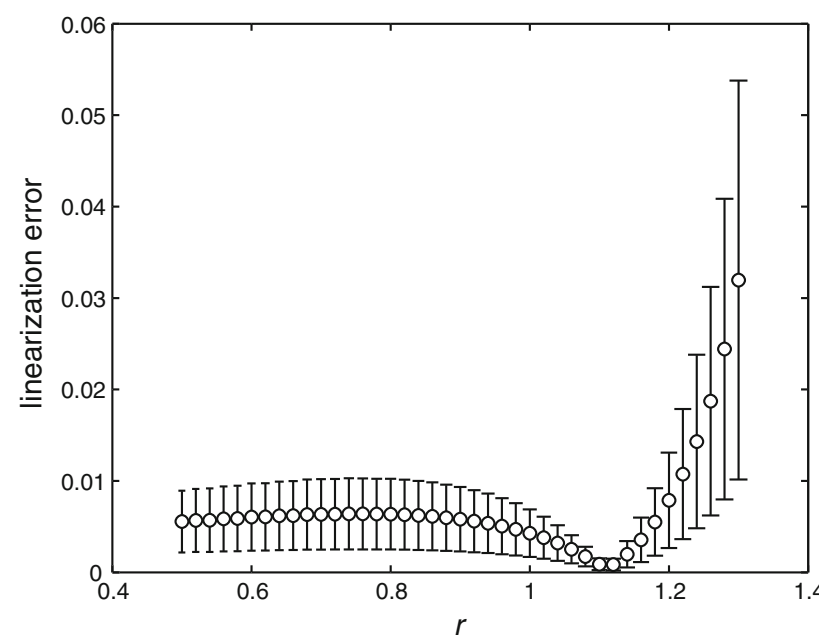

Fig. 6 Linearization errors. Linearization errors for force transmission coefficients for different ratios $r$ between the lengths of the links of the four-bar mechanism. Circles represent values averaged across opening angles. Error bars display standard deviations in the errors

not taken the internal friction force in the joints of the linkage into account. This friction force is not invariant, but a function of the opening angle $\alpha$ and the force $F_{\text {rod }}$. Therefore, the force transmission $\lambda$ in formula 5 is now replaced by a two-dimensional function of $\alpha$ and $F_{\text {rod }}$.

$F_{\text {pinch }}=\frac{1}{2} \cdot \Lambda\left(\alpha, F_{\text {rod }}\right) \cdot\left(F_{\text {pull }}-F_{\text {shaft }}\right)$

This new two-dimensional function $\Lambda\left(\alpha, F_{\text {rod }}\right)$ cannot be derived theoretically, but must be experimentally determined. To determine the effect of $\alpha$ and $F_{\text {rod }}$ on the force transmission $\Lambda$ in the presence of friction, measurements were done on a standard laparoscopic grasper. In an experimental setup, we measured the force on the rod for different opening angles and different pinch forces.

\subsection{Experimental measurement of force transmission $\Lambda$}

We measured the force on the rod of a standard laparoscopic grasper (KARL STORZ GmbH \& Co. KG, Tuttlingen, Germany) for different opening angles and different pinch forces to determine the effect of these variables on the force transmission of the linkage for the case that friction is present.

\subsection{Setup}

The handle was removed from the grasper and the shaft of the instrument was placed in a clamp that was fixed to the table (see Fig. 7). A load cell (FUTEK advanced sensor technology, INC, California, USA) was attached to the rod. The output of the load cell was recorded to a computer

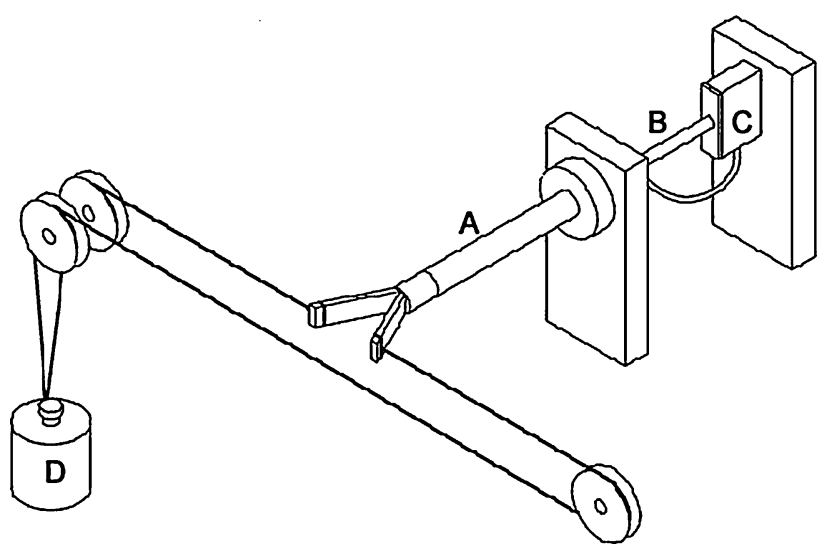

Fig. 7 Schematic drawing of the setup. Shaft A was placed in a clamp and attached to the table. The rod B was attached to the force sensor C. Mass D pulled on the wires that were attached to the tips of the forceps and guided via three pulleys

using an AD-converter (LabJack UE9, LabJack Corporation, Colorado, USA). Wires, guided by pulleys, were attached to tips of the forceps. By attaching different masses to the wires, we manipulated the forces applied on the forceps, thereby mimicking different pinch forces. We used calibrated masses of 50, 100, 150 and $200 \mathrm{~g}$, resulting in forces of $0.49,0.98,1.47$ and $1.96 \mathrm{~N}$. The opening angle could be set by translating the rod (with load cell) and fixing it when the desired opening angle was obtained. The angle between the forceps was varied between $7^{\circ}$ and $56^{\circ}$ in steps of $7^{\circ}$. These angles correspond to almost fully closing and opening the grasper.

\subsection{Procedure}

We measured the force on the rod for each combination of opening angle and mass. Each combination was repeated three times. The tests were performed in randomized order. During one measurement, the output of the load cell was recorded for a period of $1 \mathrm{~s}$ at a frame rate of $100 \mathrm{~Hz}$. The average value of this $1 \mathrm{~s}$ measurement was used for the analysis of the results. For each measurement, the grasper was first closed before applying the masses and then opened until the desired opening angle for that measurement was reached.

\section{Results}

Figure 8 displays the force on the rod for each combination of the opening angle and mass, averaged across the three repeated measurements. The transmission from the rod to the forceps for each measurement was computed using formula 3. The values thus obtained are plotted in Fig. 9.

The force transmission values that we computed for the various forces on the rod and the different opening angles 


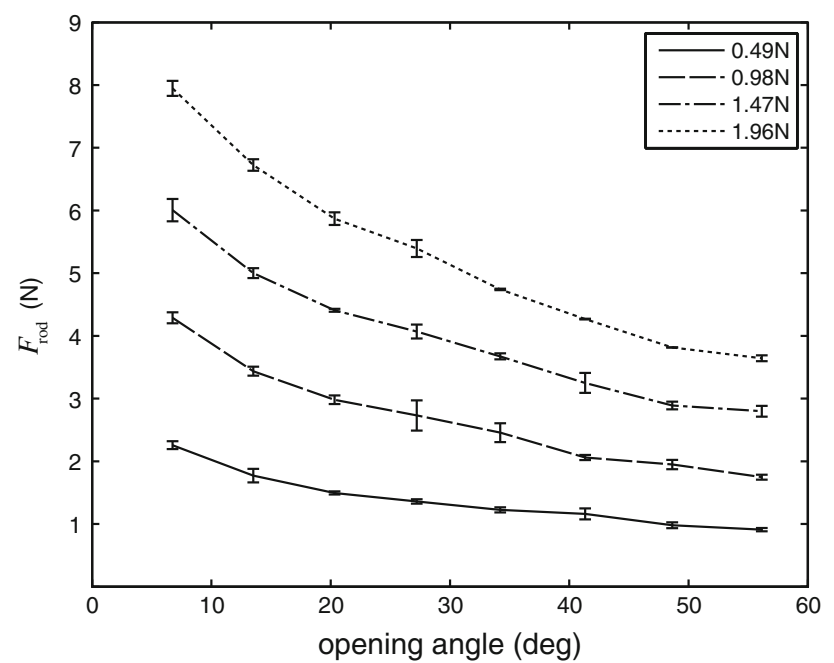

Fig. 8 Forces on the rod. Forces for the different opening angles and forces applied on the forceps. Error bars represent standard deviations around the mean for the three repeated measurements of each combination of opening angle and applied force. Opening angles represent the angle between the two forceps

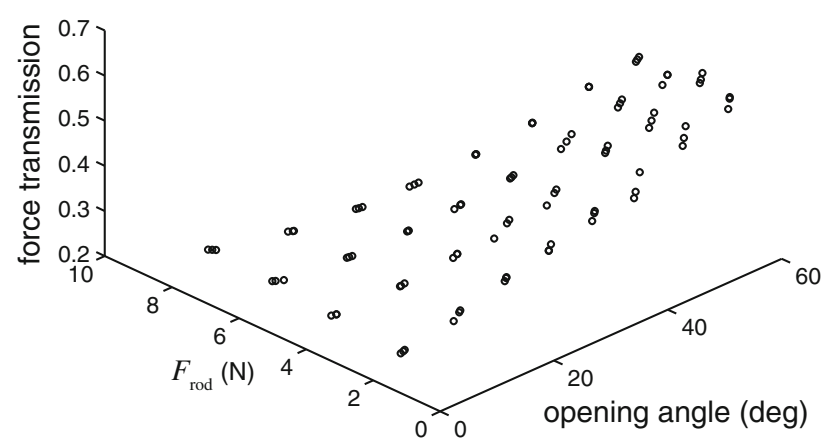

Fig. 9 Force transmission values. Transmission values for different opening angles of the grasper and different forces applied on the rod. Each circle represents a single measurement

varied between 0.22 and 0.57 . Referring to Fig. 8, we observed a clear increase in force transmission values with increasing opening angles. Increases in the force $F_{\text {rod }}$ also affect the force transmission values, but to a much lesser extent. The effect of both the opening angle and the force $F_{\text {rod }}$ appear to be roughly linear. Therefore to quantify both effects on the force transmission, we performed a multiple regression analysis to get estimates of the contribution of each component. The result of this analysis was:

$\Lambda=0.1854+0.0063 \cdot \alpha+0.0026 \cdot F_{\text {rod }}$

Equation 17 was used to predict the forces applied on the forceps from the measured values of $F_{\text {rod }}$ and the opening angles used in the experiment. In Fig. 10, the predicted forces are plotted against the forces that were actually applied in the experiment, averaged across the different opening angles. We found that the mean absolute

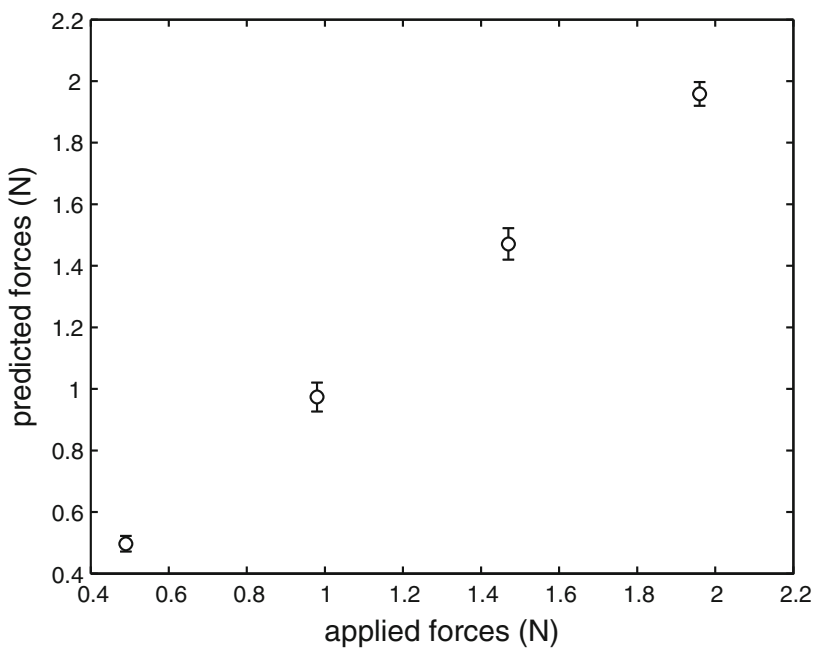

Fig. 10 Predicted forces versus applied forces. The forces on the forceps were estimated from the forces on the rod and the opening angle of the forceps using a two-dimensional linear function. The results are plotted against the actual values used in the experiment. Error bars represent standard deviations in the estimated values across opening angles

error of the predicted force was $0.03 \mathrm{~N}$ with a standard deviation of $0.02 \mathrm{~N}$.

\section{Discussion}

The analysis presented in this paper shows that it is possible to accurately determine the pulling and pinching forces of a laparoscopic grasper from force measurements at the shaft of the instrument, even when the exact geometry of the grasping mechanism is not known. This approach has the same important advantages as other indirect approaches that measure the forces at the handle of the instrument, but eases the analysis of the measurements. In an experimental study, we found that the force transmission from rod to forceps can be approximated by a twodimensional function of the forces applied on the rod and the opening angle of the grasper.

The force transmission of laparoscopic graspers varies extensively across designs due to internal friction and differences in the kinetic parameters of the linkages. The combined nonlinear contribution of these effects at the handle and forceps complicates the estimation of tissue interaction forces from measurements at the handle. Most graspers use, however, roughly the same four-bar mechanism for actuating the forceps. For such a mechanism, the force transmission from the inner rod to the forceps can be described as a sinusoidal function of the opening angle of the grasper. However, within the practical regions of opening angles of the grasper, the angular variations between the links of the four-bar mechanism are limited. 
When the grasper is fully closed, the angle that we defined as $\beta$ will be about $15^{\circ}$ and increase up to $45^{\circ}$ at the largest opening angles. Therefore, despite the nonlinear character of the force propagation through this mechanism, it can be approximated by a simple linear function of the opening angle with sufficient accuracy.

Our results show that the force transmission is not only affected by changes in opening angles, but also by the force applied on the rod. This effect is due to friction in the joints of the mechanism. These internal friction forces increase when the applied forces increase. The amount of friction will vary across designs and the materials used so that the force transmission needs to be determined empirically for each individual instrument. Furthermore, friction in the joints may cause hysteresis in the force transmission during opening and closing of the grasper. To prevent that hysteresis effects distorted our measurements the grasper was always closed first before each test. It is likely that when measurements are performed in dynamic situations one has to account for variations in the direction of motion. In that case, the force transmission needs to be determined for opening and closing movements separately.

The major benefit of the proposed method to measure tissue interaction forces is the ease of application. Only a limited number of calibration measurements are needed to generate a continuous function that enables force measurements with a high accuracy. However, we should note here that in the current study, the measurements were limited to situations where only pinch forces were applied and no pull forces. In this case, the forces on the shaft are of equal magnitude as the forces on the rod, so that only a single force sensor attached to the rod was sufficient to obtain all necessary force data. In cases where also pull forces are applied, both the force on the rod and the force on the shaft need to be measured to get a reliable estimate of the pinch and pull forces that are exerted on the tissue. One possible way to measure the forces on the shaft is the use of donut-shaped force sensors. Such commercially available circular sensors can measure the axial forces on the shaft, while the hole in the center would still allow the rod to pass through to the handle of the instrument. Further, the angular variations of mechanisms need to be measured. Although the angular changes can easily be determined from the translations of the rod, it involves implementing another sensor, thereby adding complexity and costs to the production of the instrument. Thus, even though all sensors can be placed remote from the forceps outside of the body of the patient, the proposed method requires modifications of the shaft and rod to implement the sensors. Therefore, with respect to the applicability of the approach, the issue of the design of the sensor system still needs to be addressed.
We conclude that force measurements at the shaft of laparoscopic graspers enable accurate measurements of pull and pinch forces applied on grasped tissue.

Open Access This article is distributed under the terms of the Creative Commons Attribution Noncommercial License which permits any noncommercial use, distribution, and reproduction in any medium, provided the original author(s) and source are credited.

\section{References}

1. Berkelman PJ, Whitcomb LL, Taylor RH, Jensen P (2003) A miniature microsurgical instrument tip force sensor for enhanced feedback during robot-assisted manipulation. IEEE Trans Rob Autom 19:917-922

2. De Visser H, Heijnsdijk EA, Herder JL, Pistecky PV (2002) Forces and displacements in colon surgery. Surg Endosc 16: $1426-1430$

3. Den Boer KT, Herder JL, Sjoerdsma W, Meijer DW, Gouma DJ, Stassen HG (1999) Sensitivity of laparoscopic dissectors. What can you feel? Surg Endosc 13:869-873

4. Hager-Ross C, Johansson RS (1996) Nondigital afferent input in reactive control of fingertip forces during precision grip. Exp Brain Res 110:131-141

5. Hanna GB, Drew T, Arnold A, Fakhry M, Cuschieri A (2008) Development of force measurement system for clinical use in minimal access surgery. Surg Endosc 22:467-471

6. Heijnsdijk EA, Pasdeloup A, van der Pijl AJ, Dankelman J, Gouma DJ (2004) The influence of force feedback and visual feedback in grasping tissue laparoscopically. Surg Endosc 18:980-985

7. Heijnsdijk EA, van der Voort M, de Visser H, Dankelman J, Gouma DJ (2003) Inter- and intraindividual variabilities of perforation forces of human and pig bowel tissue. Surg Endosc 17:1923-1926

8. Howe RD, Peine WJ, Kontarinis DA, Son JS (1995) Remote palpation technology. IEEE Eng Med Biol 14:318-323

9. Johansson RS, Westling G (1987) Signals in tactile afferents from the fingers eliciting adaptive motor responses during precision grip. Exp Brain Res 66:141-154

10. Kattavenos N, Lawrenson B, Frank TG, Pridham MS, Keatch RP, Cuschieri A (2004) Force-sensitive tactile sensor for minimal access surgery. Minim Invasive Ther Allied Technol 13:42-46

11. Payandeh S, Fraser S (1997) Force propagation models in laparoscopic tools and trainers. Proc IEEE Eng Med Biol Soc Int Conf 3:957-960

12. Peirs J, Clijnen J, Reynaerts D, van Brussel H, Herijgers $\mathrm{P}$, Corteville B, Boone S (2004) A micro optical force sensor for force feedback during minimally invasive robotic surgery. Sens Actuators A 115:447-455

13. Sjoerdsma W, Herder JL, Horward MJ, Jansen A, Bannenberg JJG, Grimbergen CA (1997) Force transmission of laparoscopic grasping instruments. Minim Inv Ther Allied Technol 6:274-278

14. Tholey G, Desai JP (2008) A compact and modular laparoscopic grasper with tridirectional force measurement capability. J Med Devices 2:031001.1-0310010.8

15. Trejos AL, Patel1 RV, Naish MD (2010) Force sensing and its application in minimally invasive surgery and therapy: a survey. Proc Inst Mech Eng, J Mech Eng Sci 224:1435-1454 